\title{
Extinction versus counterconditioning for the meaning of words and nonsense syllables
}

ADAM MILLER, MICHAEL GIMPL, and RUSSELLL MCCRIMMON, Saint Cloud State College, St. Cloud, Minn. 56301

This study analyzed the effects of extinction, counterconditioning and the number of trials of each upon the degree of rated meaning for words and nonsense syllables. For 200 Ss counterconditioning was effective, but extinction and trials were not, in changing meaning. Awareness was unrelated to meaning change.

Perhaps prematurely, Staats (1968) projected to theoretical significance the process he termed the higher-order classical conditioning of word meaning. Experimental investigations have failed to support fully Staats' conceptualization of the process. Contrary evidence from several experiments, e.g., Cohen (1964), indicated that only aware Ss changed word meaning. In other evidence, pertinent here, extinction failed to change word meaning (Insko \& Oakes, 1966; Miller \& Barsness, in press) and counterconditioning also failed (Das \& Mitra, 1965). The present study contrasted extinction and counterconditioning procedures for changing the meaning of words and nonsense syllables.

\section{METHOD}

Two hundred undergraduate volunteers were assigned to eight groups of $25 \mathrm{Ss}$ each. All Ss responded through these six phases: preassociation rating, paired-associate learning, postassociation rating, change learning, postchange rating, and awareness measurement.

The rating sheet used for all rating phases contained four nonsense syllables (NS) and 11 meaningful words(MW). Ss rated each on the $\operatorname{good}(=1) \cdot \operatorname{bad}(=7)$ semantic differential scale. Included were the NSs KUJ and XAD and the MWs RIGID and DARK. The former were selected for low-association norm values and the latter for neutral good-bad rating norm values.

There were two sets of paired-associate lists, each containing 16 pairs. In one set the stimulus word XAD was paired separately with each of the eight MWs of good meaning (1.30 to 1.57 on the good-bad scale) based on the Jenkins et al (1958) norms and the stimulus word KUJ was paired with each of eight words of bad meaning (6.37 to 6.67). The 16 NS-MW pairs were randomly ordered to form each list. For the second set of lists the MW RIGID replaced XAD and DARK replaced KUJ.
In paired-associate learning the stimulus word was projected on a screen in the experimental classroom for $4 \mathrm{sec}$ and $E$ said the listed MW which all Ss repeated aloud. The interpair interval was 1 sec. Two random orders of the list were presented followed by a memory test.

In the fourth phase of experimentation both change procedures used pairedassociate learning as described above. For counterconditioning the MWs originally associated with $X A D$ were now associated with KUJ and those for RIGID and DARK were reversed. For extinction the stimulus words were presented with no associated MWs. Depending upon the experimental condition either one list or six lists were presented.

Finally, Ss answered a 20-item multiplechoice questionnaire to measure awareness factors identified by Miller (1968). Two major factors were awareness that (1) the eight MWs associated with a stimulus word had common meaning and (2) one purpose of the experiment was to change the meaning of stimulus words.

In a 2 by 2 factorial design, one independent variable was the kind of stimulus word (NS or MW) and a second was the number of list presentations used in change learning (one or six). The major dependent variables were ratings of NSs and MWs and awareness scores.

According to Staats (1968) classical conditioning may be assumed for the above procedures; the stimulus words are CSs. The MWs with common meaning are UCSs. The meaning common to the eight MWs that are the associates to a single stimulus word is a physiological response which is the UCR. The meaning response mediates the rating of the stimulus word.

\section{RESULTS}

To test the effects of paired-associate learning on ratings, changes from preassociation to postassociation were compared separately by analysis of variance for NSs and MWs. The mean values changed for $K U J$ from 4.50 to $5.49(+0.99)$ and for XAD from 4.60 to $2.86 \quad(-1.74)$ $[F(3,1062)=12.82, p<.01]$. The corresponding changes for DARK were 3.55 to $4.52(+0.97)$ and for RIGID were 4.58 to $3.61(-0.97)[F(3,1062)=2.67, p<.05]$ All ratings changed toward the values of the MW associates.

Comparison between the effects of extinction and counterconditioning involved analyzing changes from postassoci- ation to postchange ratings, separately tor NSs and MWs. For counterconditioning of NSs, KUJ changed from 5.18 to 2.48 $(-2.70)$ and XAD changed from 2.88 to $5.64(+2.76)$, both in the direction of their MW associates. For extinction the corresponding changes were 5.80 to $5.54(-0.26)$ and 2.84 to $2.98(+0.14)[\mathrm{F}(1,96)=5.53$, $\mathrm{p}<.05]$. Counterconditioning effectively changed the rated meaning of NSs but extinction did not. For MWs both counterconditioning and extinction resulted in nonsignificant changes in rated meaning $[F(1,96)=0.11, p>.05]$.

The number of trials for extinction and counterconditioning, whether one or six, had no significant relationship to the degree of change in rated meaning. To check for generalization of original meaning change and of counterconditioning, NSs similar in number of letters (KUQ for KUJ and XAZ for XAD) and similar MWs (HARD for RIGID and DIM for DARK) were included in the ratings. None of the changes was statistically significant.

The final analysis compared the original meaning change with Ss' awareness. Ss were classified in two ways: Those who changed ratings of KUJ and XAD or RIGID and DARK appropriately vs those who did not, and those who were aware vs those whowere unaware on each of two kinds of awareness. For NSs, when awareness of the experimental purpose was compared to actual change in ratings, chi square $=0.51, \mathrm{df}=1$ for six items combined. A similar comparison for awareness that MW associates had common meaning gave a chi square $=3.41, \mathrm{df}=1$ for four items combined. For MWs the corresponding chi squares were 2.40 and 2.04. None was significant at $p<.05$. For 10 awareness items $40 \%$ of the Ss who changed their ratings also were aware (both kinds). Awareness was not necessary for meaning change.

\section{DISCUSSION}

The above results combined with those of Miller \& Barsness (in press), Insko \& Oakes (1966) and others, only partially fit Staats' (1968) conceptualization of meaning change as classical conditioning. Application of meaning change procedures to nonexperimental situations will be seriously limited if replication confirms that counterconditioning has different effects for MWs and NSs. Meaning change appears to be in part an automatic association process but one not necessarily dependent upon Ss' awareness. 


\section{REFERENCES}

COHEN, B. H. Role of awareness in meaning established by classical conditioning. Journal of Experimental Psychology, 1964, 67, 373-378. DAS, J. P., \& MITRA, A. K. Persistence of acquired meaning in semantic conditioning. Psychonomic Scienœ, 1965, 3, 83-84.

INSKO, C. A., \& OAKES, W.F. Awareness and the "conditioning" of attitudes. Journal of Personality \& Social Psychology, 1966, 4, 487-496.

JENKINS, J. J., RUSSELL, W. A., \& SUCI, G. J.
An atlas of semantic profiles for 360 words. American Journal of Psychology, 1958, 71, 688-699.

MILLER, A. W., JR. The dimensionality of awareness in verbal conditioning. Journal of Psychology, 1968, 70, 99-110.

MILLER, A. W., JR., \& BARSNESS, W Extinction, stimulus generalization and partial reinforcement for higher-order meaning conditioning. Psychological Reports, in press.

STAATS, A. W. Learning, language and cognition. New York: Holt, Rinehart \& Winston, 1968.

\section{Lateral eye movement, reading speed, and visual attention}

\section{PAUL BAKAN and R. LANCE SHOTLAND, Michigan State University, East Lansing, Mich. 48823}

An $S$, looking at the eyes of a questioner, will make a lateral eye movement, consistently right or left, as he begins to reflect on the answer to the question. To test the hypothesis that right movers do better at tasks requiring visual attention, the Stroop color-word interference test was administered to two groups, right and left movers. Right movers were subject to significantly less interference than left movers, thus confirming the hypothesis. Right movers were also found to read faster a list of color names printed in black on white. It is suggested that slower reading time on the color-word card is due in part to the covert response of reading the word, and that the better performance of right movers is due to their greater facility in the making of this covert reading response.

Day (1964) has described a lateral eye movement response which occurs when an individual shifts his attention from an external to an internal focus. If a person's visual attention is directed toward your face while you ask him a question requiring reflection, the eye movement occurs when you finish talking and he begins to think of an answer. It can be observed by watching one or both eyes as he shifts from external to internal attention in his search for an answer to the question. The movement is characteristic in direction for most individuals on repeated observations (Day, 1964; Duke,
1968). The lateral eye movement is most likely to occur in response to complex or reflective questions; simple factual questions are less likely to elicit the movement.

Day $(1964,1967 a, b)$ has made a number of clinical observations and speculations about the phenomenon suggesting that the direction of lateral movement should have correlates in a variety of personality, cognitive, and physiological variables. On the basis of his observations Day suggests that "in general the right mover shows an externalized actively responsive distribution of attention emphasizing the visual-haptic modes," whereas "the left mover shows an internalized, subjective ... distribution of attention in which he is more reactive to auditory and subjective visceral experience [Day, 1967a]."

This study was designed to test the hypothesis that Ss who make right lateral eye movements will perform better than those who make left lateral eye movements in a task requiring a high degree of visual attention. Right and left movers were compared for performance on the Stroop color-word test (Stroop, 1935, 1938). In this test, $S$ is presented with color words (e.g., red, blue, yellow, green) printed in conflicting ink colors. Thus, the word "red" is printed in green ink and the correct response is saying the word "green." This test has been used as a measure of visual attentiveness or resistance to distraction (Agnew \& Agnew, 1963; Bakan \& Alperson, 1967; Klein, 1964; Lazarus, Baker, Broverman, \& Moyer, 1957). The literature on the test has been reviewed by Jensen (1965) and Jensen \& Rohwer (1966).
SUBJECTS

A total of 53 Ss (29 male and 24 female) were tested. All were university students from various fields of study at levels ranging from freshmen to graduate students. COLOR-WORD TEST

Three $9 \times 12$-in. cards were presented in the same sequence to all $S s$, a word card (w), a color card (c), and a color-word card (cw).

The w card consisted of 80 color names (red, blue, green, yellow) printed in black, in 10 rows of 8 . The words were randomly ordered with the restriction that no word courd follow itself. The task for $S$ was to read the names as rapidly as possible without pointing to the words and to correct all errors.

The c card consisted of 80 colored rectangular patches (.15 x .50 in.) of red, green, blue, or yellow ink. These were ordered randomly with the restriction that no color could follow itself in any row. The $S$ was instructed to name the colors as rapidly as possible without pointing to the colors and to correct all errors.

The cw card consisted of the words red, blue, green, and yellow printed in colors different from that of the word; thus, the word "red" might appear in ink of either blue, green, or yellow. The order of the ink colors was identical to the order of the colors on the c card. The order of color names was the reverse order of the color names on the w card. The Ss were instructed to name the colors in which the words were printed as rapidly as possible without pointing to the word, squinting, or blurring the word. They were instructed to correct all errors.

\section{PROCEDURE}

Each $S$ sat in a chair facing $E$ seated $4 \mathrm{ft}$ away. The $S$ was asked to solve mental arithmetic problems such as $123 / 5 \times 4$, $18 \times 5,136 / 6 \times 5,16 \times 5$, etc. The $E$ watched the right eye and recorded the initial lateral eye movement following the statement of the problem. If the first lateral movements for four consecutive questions went in the same direction $S$ was classified as either a right of left mover. Generally, more than four questions had to be presented as some of the questions were not sufficiently complex to elicit an eye movement. If $S$ shut his eyes or turned away from $E$, he was told to keep his eyes open and face $E$, though it was not necessary to stare at $E$ while solving the problems.

After $S$ had been classified as a right or left mover the color-word test was administered. Cards were presented and performance was timed in the following order: w card, c card, cw card.

\section{RESULTS}

Of the 53 Ss there were 49 who could be classified as either right or left movers. Of these, 28 were right movers and 21 were left movers. One was etiminated because of color 